\title{
Tunneling in Suspended Carbon Nanotubes Assisted by Longitudinal Phonons
}

\author{
S. Sapmaz, P. Jarillo-Herrero, Ya. M. Blanter, C. Dekker, and H. S. J. van der Zant \\ Kavli Institute of Nanoscience, Delft University of Technology, P.O. Box 5046, 2600 GA Delft, The Netherlands
}

(Received 8 August 2005; published 19 January 2006)

\begin{abstract}
Current-voltage characteristics of suspended single-wall carbon nanotube quantum dots show a series of steps equally spaced in voltage. The energy scale of this harmonic, low-energy excitation spectrum is consistent with that of the longitudinal low- $k$ phonon mode (stretching mode) in the nanotube. Agreement is found with a Franck-Condon-based model in which the phonon-assisted tunneling process is modeled as a coupling of electronic levels to underdamped quantum harmonic oscillators. A comparison with this model indicates a rather strong electron-phonon coupling factor of order unity.
\end{abstract}

PACS numbers: 73.63.Fg, 73.23.Hk

In nanoelectromechanical systems, mechanical motion affects electrical current and vice versa [1-4]. Of special interest is the study of electron-phonon coupling in these devices since tunneling of a single electron may induce a displacement of the movable structure [5-10]. The interaction strength is characterized by the dimensionless electron-phonon ( $e$-ph) coupling constant $g$, which is proportional to the ratio of the classical and the quantum displacement. In bulk systems the $e$-ph coupling is generally weak and the coupling constant is orders of magnitude smaller than 1 . However, since the coupling dramatically increases with decreasing device mass, nanoelectromechanical devices may exhibit an intermediate to strong $e$-ph coupling [11-13]. In this regime, current-voltage characteristics are expected to exhibit additional steps whose height can be used as an estimate of $g$. For example, $g$ is around one in the $\mathrm{C}_{60}$ molecular devices of Ref. [11], while measurements on different $\mathrm{C}_{140}$ samples [12] indicate a value of $g$ between 0.2 and 8 .

Carbon nanotubes are ideal systems for exploring electromechanical effects since they have a small diameter, a low mass, and can be defect free on a molecular level. In experiments on suspended nanotubes, different methods have already been used to probe the bending $[14,15]$ and radial breathing mode (RBM) [16]. The measurements show that the free-hanging tubes operate in the underdamped regime of low dissipation. For the fundamental bending mode the reported quality factor is about 100; for the RBM it is estimated as high as 10000 .

In this Letter we present electronic transport spectroscopy measurements on suspended single-wall nanotubes, which show signatures of phonon-assisted tunneling, evidenced by the presence of a series of steps in the $I-V$ characteristics. Such steps form a harmonic low-energy spectrum, whose energy scale and length dependence are consistent with that of the longitudinal stretching mode. A comparison with the Franck-Condon theory shows that the $e$-ph coupling constant is of order 1 .

Devices are fabricated by locating individual nanotubes (laser ablation and chemical vapor deposition) on a $\mathrm{Si} / \mathrm{SiO}_{2}$ substrate using an atomic force microscope
(AFM) with respect to predefined markers. Subsequently, the electrodes are made using conventional $e$-beam lithography techniques and thermal evaporation of $\mathrm{Cr}(5 \mathrm{~nm})$ and $\mathrm{Au}(50 \mathrm{~nm})$. The nanotubes are suspended by removing the underlying $\mathrm{SiO}_{2}$ in a wet etch step using buffered HF [17]. A schematic sample geometry and a scanning-electron microscope micrograph are shown in Fig. 1. In the experiment the source and gate voltage are defined with respect to the drain, which is connected to ground.

In Fig. 2 we show stability diagrams for three nanotubes measured at (a) $10 \mathrm{mK}$ and (b),(c) $300 \mathrm{mK}$ where the differential conductance, $d I / d V$, is plotted versus bias and gate voltage. The three metallic nanotubes have a length between source and drain contacts, $L$, ranging from 0.14 to $1.2 \mu \mathrm{m}$. Their diameter $d$ is between 1 and $1.4 \mathrm{~nm}$ as determined from AFM imaging. In the diamond shaped regions (Coulomb diamonds) the current is zero due to Coulomb blockade, and the charge number in the dot is fixed. Regular and closing Coulomb diamonds indicate single dot behavior $[18,19]$ in all three samples for the gate range shown. Notice that the diamonds in Fig. 2(a) do close, as shown in the inset, which was taken at a higher temperature $(300 \mathrm{mK})$ in a different cooldown. The low-bias current, however, is suppressed, which
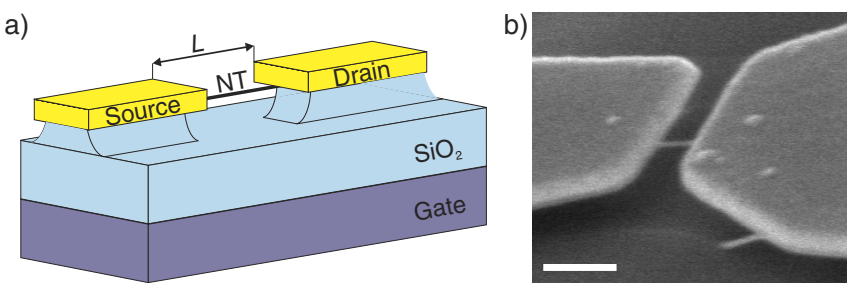

FIG. 1 (color online). (a) Schematic drawing of a suspended nanotube (NT) clamped between two $\mathrm{Cr}$ /Au electrodes on top of silicon oxide. The underlying oxide is partially removed by a wet etch step leaving the nanotube suspended. The highly doped silicon plane is used as a global gate to tune the electrostatic potential of the nanotube. (b) Scanning-electron microscope micrograph of a suspended nanotube. The scale bar represents $200 \mathrm{~nm}$. 

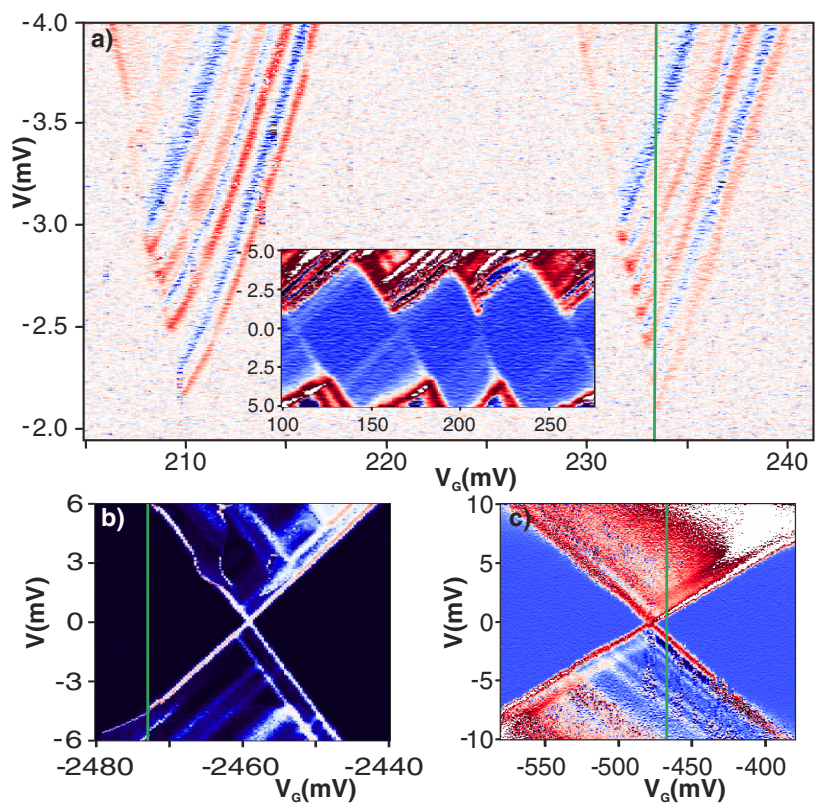

FIG. 2 (color online). Stability diagrams for three different suspended nanotubes with a length in between contacts of $1.2 \mu \mathrm{m}, 420 \mathrm{~nm}$, and $140 \mathrm{~nm}$ for (a), (b), and (c), respectively. The conductance $(d I / d V)$ is plotted as a function of source-drain voltage, $V$, and gate voltage, $V_{G}$. Blue corresponds to low and red to high conductance. Measurements have been performed at $T=300 \mathrm{mK}$ except in (a), where the base temperature was $10 \mathrm{mK}$. (a) Small region of a stability diagram showing closely spaced sets of lines running parallel to the Coulomb diamond edges for two charge states. At low bias, a strong suppression of the conductance is present. Red lines indicate positive differential conductance; blue lines indicate negative differential conductance. Inset: regular diamonds that close are observed in a different cooldown at $T=300 \mathrm{mK}$. (b), (c) Diamond crossings for two other samples, again showing lines parallel to the diamond edges with energy separations smaller than expected for electronic excitations.

could be a signature of strong electron-phonon coupling $[5,9,10]$.

Excitations of a quantum dot appear as lines running parallel to the Coulomb diamond edges in the stability diagrams [18]. At such a line, a new electronic level becomes resonant with the leads and an additional transport channel opens up. The energy of an excitation can be determined by reading off the intersection point between the excitation line and the Coulomb diamond edge on the bias axis [19]. Furthermore, the excitations correspond to the charge state of the Coulomb diamond they end up in. Electronic excitations in nanotubes typically differ between adjacent charge states [20]. In Fig. 2(a), a dense set of equally spaced excitation lines (starting from the first electronic excitation) is clearly visible near $\mathrm{V}_{G}=210$ and $230 \mathrm{mV}$; i.e., adjacent charge states exhibit a similar set of excitations with approximately the same energy spacing. The fact that excitations occur primarily in one direction is due to asymmetric tunnel barriers [19].

The energy differences between the excitation lines of Fig. 2 are shown in the insets of Fig. 3. In all three cases, the excitation energy is an integer multiple of the first (fundamental) excitation. Thus, they form a harmonic spectrum with up to five levels. A linear fit yields an excitation energy of 140,690 , and $530 \mu \mathrm{eV}$ for the tubes with length (a) $1.2 \mu \mathrm{m}$, (b) $420 \mathrm{~nm}$, and (c) $140 \mathrm{~nm}$, respectively. These values are an order of magnitude smaller than the expected mean electronic level spacing given by $\Delta=h v_{F} / 2 L$, with $h$ the Planck constant and $v_{F}=8.1 \times 10^{5} \mathrm{~m} / \mathrm{s}$ [21] the Fermi velocity.

A more natural explanation for the observed harmonic spectra is a vibrational mode coupled to electron tunneling [11]. Multiple steps with identical spacing would then arise from the excitation of an integer number of vibrational quanta. Indeed, the observed equidistant energy separation is consistent with that expected from the longitudinal stretching mode in the nanotubes. In Fig. 4, we plot the energy of important low-energy vibrational modes of single-wall nanotubes [22,23]. For comparison, we plot the mean electronic energy level separation, $\Delta$, in black. The (blue) squares correspond to the fundamental vibrational excitation energy extracted from the linear fits in the insets of Fig. 3. The energy of the radial breathing mode (green) does not depend on the nanotube length and equals
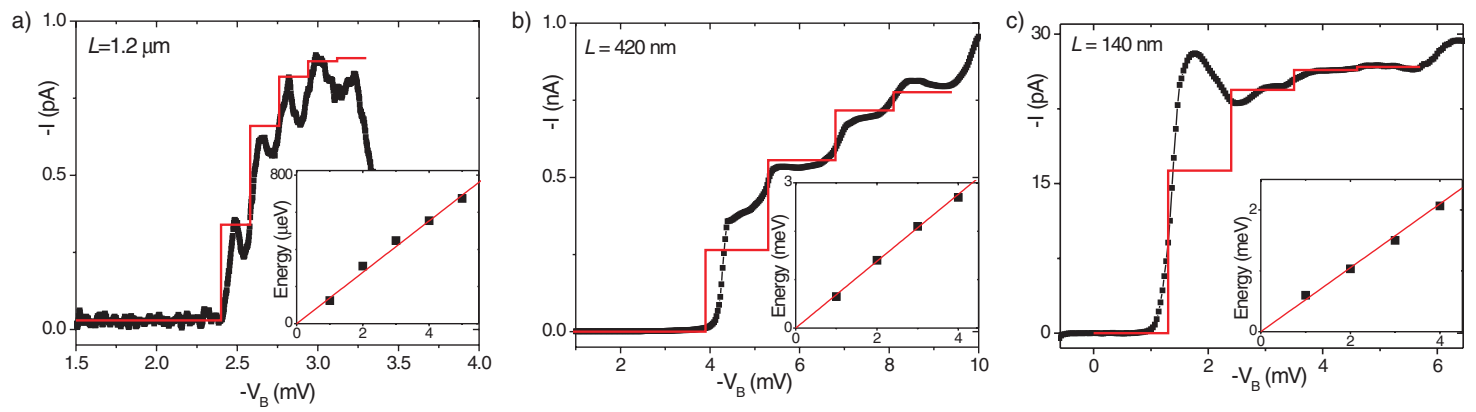

FIG. 3 (color online). Current as a function of source-drain voltage at a voltage indicated by the vertical (green) lines in Fig. 2. The drawn (red) lines represent the step heights calculated in the Franck-Condon model (see text) for an electron-phonon coupling parameter of $0.95,1.1$, and 0.5 for (a), (b), and (c), respectively. In the insets, the energy separation between the peaks or steps (lines in Fig. 2) is plotted, showing an equally spaced, harmonic spectrum. The slope of the drawn line is 140,690 , and $530 \mu \mathrm{eV}$ for the insets of (a), (b), and (c), respectively. 


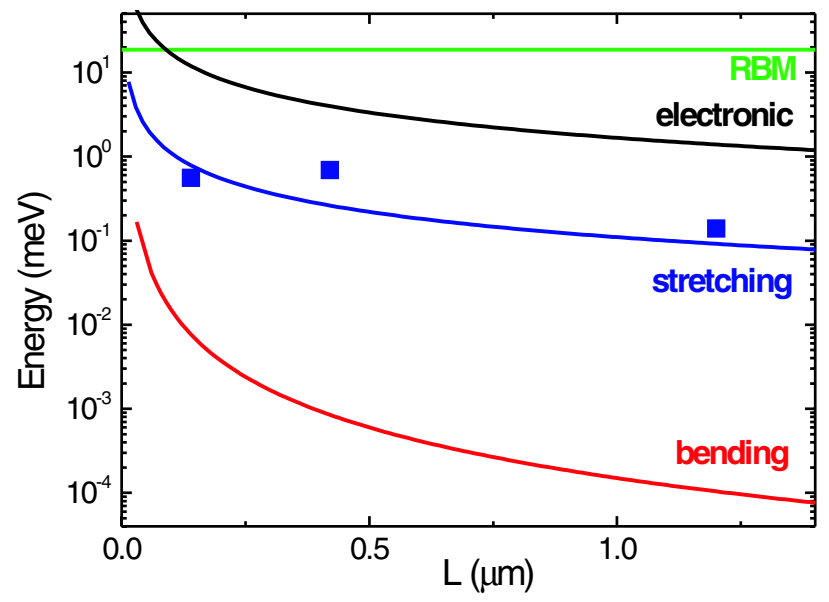

FIG. 4 (color online). Energy scales of different vibrations and electronic excitations plotted on a log scale for a nanotube with a $1.4 \mathrm{~nm}$ diameter. The radial breathing mode (green) does not depend on the length $L$. The bending mode vibrations (red) have a $L^{-2}$ dependence. The mean electronic level spacing (black) and the stretching mode (blue) vibrations depend inversely on the length.

$28 \mathrm{meV} / d(\mathrm{~nm})$. The bending mode (red) has a $L^{-2}$ dependence [24] and an energy much smaller than the measured excitation energy. The stretching mode vibration energy (blue) is inversely proportional to the length [25], $E=$ $(n h / L) \sqrt{Y / \rho_{m}}$, where $Y$ is Young's modulus, $\rho_{m}$ is the density, and $n$ is the vibrational quantum number. For nanotubes with $\rho_{m}=1.3 \mathrm{~g} / \mathrm{cm}^{3}, Y=1 \mathrm{TPa}$ the vibrational energy corresponding to the fundamental mode is $\sim 110 \mu \mathrm{eV} / L(\mu \mathrm{m})$ [23]. As Fig. 4 shows, the data are in good agreement with these predicted values.

The coupling of electronic levels with vibrational modes (quantum harmonic oscillators) can be described in terms of the Franck-Condon model [26]. According to the Franck-Condon principle, an electron in an electronic transition moves so fast that the nuclear positions are virtually the same immediately before and after the transition. As a consequence, the transition rate is proportional to the Franck-Condon factors defined as the square of the overlap integral between the vibrational wave functions of the two states involved. An important parameter is the electronphonon coupling factor, $g=\frac{1}{2}\left(\frac{x}{x_{0}}\right)^{2}$. This is the ratio of the classical displacement length, $x$, to the quantum mechanical oscillator length, $x_{0}=\sqrt{\hbar / m \omega}$. Alternatively, $g=$ $\frac{F^{2}}{2 \hbar m \omega^{3}}$, where $F$ is the force on, $m$ the mass of, and $\omega$ the frequency of the oscillator.

For low damping, the vibrational levels remain sharp and the Franck-Condon model predicts steps in the currentvoltage characteristics that are equally spaced in energy (bias voltage). In the presence of strong relaxation, the normalized step heights are given by [5] $P_{n}=e^{-g} g^{n} / n$ !. In the strong coupling $(g \gg 1)$ limit, the height of the first steps is exponentially suppressed (phonon blockade) $[5,9,10]$. Multiple steps arise only if $g$ is of the order of 1 or larger, and the observation of a spectrum of equally spaced excitation lines therefore indicates that the $e$-ph coupling in our suspended nanotubes must be rather strong.

In Fig. 3, the calculated (red) curves represent the step heights $\left(P_{n}\right)$ given by the Franck-Condon model with strong relaxation discussed above. The symbols are the experimental curves taken at the vertical (green) lines in Fig. 2. Considering the simplicity of the model, reasonable agreement is obtained in all three cases. The comparison yields an estimate of $g$ of $0.95,1.1$, and 0.5 in Figs. 3(a)3 (c) respectively, indicating that it is approximately length independent. We have also performed a similar analysis at other gate voltages yielding the same $g$ values.

The theoretical curves in Fig. 3 do not exactly follow the measured ones. Better fits may be obtained if the influence of a gate voltage and asymmetric coupling is considered [5] or if coupling to excited electronic states [27] is considered or if the influence of damping or nonequilibrium phonons (weak relaxation) is taken into account. In the latter case the peak heights are expected to display a nonsystematic dependence on $g$ and peak number [9]. Consideration of these effects is, however, beyond the scope of this Letter.

The high value of the $e$-ph coupling $(g \sim 1)$ is remarkably since in graphite the coupling between electrons and longitudinal phonons is weak. A source of intermediate to strong coupling could be the interaction between longitudinal and transverse vibrations [28]. Alternatively, we find that in suspended nanotubes the same $e$-ph coupling mechanism as in the bulk [29] can lead to a $g \sim 1$ if the electron density is inhomogeneous. The calculation proceeds as follows: The interaction energy of electrons with the polarization charge is characterized by the energy

$$
W=-\int d x d x^{\prime} \rho(x) K\left(x-x^{\prime}\right) \frac{\partial P}{\partial x^{\prime}} .
$$

Here, $\rho(x)$ is the density of excess charge produced by one electron, $K\left(x-x^{\prime}\right)$ is an interaction kernel, which we approximate by $\delta\left(x-x^{\prime}\right)$ for the case that interactions are effectively screened by the gate, and $P(x) \approx e \rho_{0} z(x)$ is the polarization vector. The parameter $\rho_{0}$ is the ion density and $z(x)$ is the displacement, which in the singlemode approximation becomes $z(x)=A_{n} \sin (\pi n x / L)$. Calculating the force $F=-\partial W / \partial A_{n}$, we obtain

$$
F=\frac{e \rho_{0} \pi n}{L} \int_{0}^{L} d x \rho(x) \cos \frac{\pi n x}{L} .
$$

If the excess charge density is uniform, $\rho(x)=e / L, F=0$ for all modes. The incorporation of interactions that are screened at distances longer than the distance to the gate yields a force that scales as $L^{-2}$. In this case, the coupling parameter $g$ also scales as $L^{-2}$ and typical values are in the order of $10^{-3}$, in apparent contradiction with the experimental data.

Assuming that the charge is localized in the center of the tube, $\rho(x)=e \delta(x-L / 2)$, the force is zero for odd har- 
monics, but for even harmonics, $n=2 l$, it reads $F_{l}=$ $(-1)^{l} e^{2} \rho_{0} 2 \pi l / L$. This results in a coupling parameter that is length independent and scales as $l^{-1}$ : higher modes are coupled weaker to electrons. Numerical estimates show that $g \sim 1$. Localization of an electron in a point away from the middle produces coupling to both odd and even modes. Note that the electron does not have to be strongly localized to produce a $g \sim 1$. Such a nonuniform density can be created by impurities located in the substrate or induced by a redistribution of electrons in a suspended tube bent by a underlying gate electrode [30].

An interesting feature of the data is the appearance of negative differential conductance (NDC) in the currentvoltage characteristics. NDC is very pronounced in Fig. 3(a), but is also present in Figs. 3(b) and 3(c). Although several explanations for NDC have been put forward, its origin remains unclear. Koch and von Oppen [10] showed that for low relaxation and strong $e$-ph coupling, NDC features appear, although they do not follow regions with strong positive differential conductance (PDC) as in our data. McCarthy et al. [6] have shown that NDC features can be due to an $e$-ph coupling that is voltage dependent. Their calculations also reproduce the catastrophic current decrease of Fig. 3(a) for bias voltages higher than $3 \mathrm{mV}$. However, at the moment we do not know how such a voltage dependence would arise in suspended tubes. Finally, Nowack and Wegewijs [27] have considered a Franck-Condon model with a coupling to an electronic ground state and its excited state. They show that the competition between the two states generates strong NDC effects. NDC and PDC lines may have the same gate voltage dependence preceded by a region of suppressed current. This scenario may especially be relevant for the data in Fig. 3(a).

In summary, transport measurements on suspended single-wall carbon nanotubes show signatures of phononassisted tunneling, mediated by longitudinal vibrational (stretching) modes. The current-voltage characteristics show multiple steps whose heights are in reasonable agreement with the Franck-Condon predictions if the $e$-ph coupling constant is of order unity. Suspended nanotube quantum dots form an interesting model system for future studies on the interaction between single electrons and quantized phonons in the intermediate to strong electronphonon coupling limit.

We thank Karsten Flensberg, Wataru Izumida, Milena Grifoni, Leo Kouwenhoven, Leonid Gurevich, and Maarten Wegewijs for discussions. Financial support is obtained from the Dutch organization for Fundamental Research on Matter (FOM), which is financially supported by the "Nederlandse Organisatie voor Wetenschappelijk Onderzoek" (NWO), and this research was supported by EC FP6 funding (Contract No. FP6-2004-IST-003673). This publication reflects the views of the authors and not necessarily those of the EC. The Community is not liable for any use that may be made of the information contained herein.

[1] M. L. Roukes, Phys. World 14, 25 (2001).

[2] A. N. Cleland, Foundations of Nanomechanics (Springer, Berlin, 2002).

[3] L. Y. Gorelik et al., Phys. Rev. Lett. 80, 4526 (1998).

[4] A. Erbe, C. Weiss, W. Zwerger, and R. H. Blick, Phys. Rev. Lett. 87, 096106 (2001).

[5] S. Braig and K. Flensberg, Phys. Rev. B 68, 205324 (2003).

[6] K. D. McCarthy, N. Prokof'ev, and M. T. Tuominen, Phys. Rev. B 67, 245415 (2003).

[7] Ya. M. Blanter, O. Usmani, and Yu. V. Nazarov, Phys. Rev. Lett. 93, 136802 (2004); 94, 049904(E) (2005).

[8] N. M. Chtchelkatchev, W. Belzig, and C. Bruder, Phys. Rev. B 70, 193305 (2004).

[9] A. Mitra, I. Aleiner, and A. J. Millis, Phys. Rev. B 69, 245302 (2004).

[10] J. Koch and F. von Oppen, Phys. Rev. Lett. 94, 206804 (2005).

[11] H. Park et al., Nature (London) 407, 57 (2000).

[12] A. N. Pasupathy et al., Nano Lett. 5, 203 (2005).

[13] E. M. Weig et al., Phys. Rev. Lett. 92, 046804 (2004).

[14] B. Reulet et al., Phys. Rev. Lett. 85, 2829 (2000).

[15] V. Sazonova et al., Nature (London) 431, 284 (2004).

[16] B. J. LeRoy, S. G. Lemay, J. Kong, and C. Dekker, Nature (London) 432, 371 (2004).

[17] J. Nygård and D. H. Cobden, Appl. Phys. Lett. 79, 4216 (2001).

[18] Single Charge Tunneling, edited by H. Grabert and M. H. Devoret (Plenum, New York, 1992).

[19] L.P. Kouwenhoven, D. G. Austing, and S. Tarucha, Rep. Prog. Phys. 64, 701 (2001).

[20] D. H. Cobden, M. Bockrath, P. L. McEuen, A. G. Rinzler, and R.E. Smalley, Phys. Rev. Lett. 81, 681 (1998).

[21] S. Lemay et al., Nature (London) 412, 617 (2001).

[22] M. S. Dresselhaus and P. C. Eklund, Adv. Phys. 49, 705 (2000); H. Suzuura and T. Ando, Phys. Rev. B 65, 235412 (2002); A. De Martino and R. Egger, Phys. Rev. B 67, 235418 (2003); W. Izumida and M. Grifoni, New J. Phys. 7, 244 (2005).

[23] The twisting mode vibration of the nanotube has a comparable energy with the stretching mode. However, the twisting mode couples much less effectively than the stretching mode [22].

[24] S. Sapmaz, Ya. M. Blanter, L. Gurevich, and H. S. J. van der Zant, Phys. Rev. B 67, 235414 (2003).

[25] L.D. Landau and E.M. Lifshitz, Theory of Elasticity (Pergamon, Oxford, 1986).

[26] G.C. Schatz and M. A. Ratner, Quantum Mechanics in Chemistry (Prentice-Hall, Englewood Cliffs, 1993).

[27] K. C. Nowack and M. Wegewijs, cond-mat/0506552.

[28] K. Flensberg (private communication).

[29] A. A. Abrikosov, Fundamentals of the Theory of Metals (North-Holland, New York, 1988).

[30] D. Fedorets (unpublished). 